\title{
Vibrational signatures for the identification of single-photon emitters in hexagonal boron nitride
}

\author{
Christopher Linderälv, ${ }^{1}$ Witlef Wieczorek $\odot,{ }^{2}$ and Paul Erhart $\oplus^{1, *}$ \\ ${ }^{1}$ Chalmers University of Technology, Department of Physics, Gothenburg 41296, Sweden \\ ${ }^{2}$ Chalmers University of Technology, Department of Microtechnology and Nanoscience, Gothenburg 41296, Sweden
}

(Received 13 August 2020; revised 13 January 2021; accepted 17 February 2021; published 15 March 2021)

\begin{abstract}
Color centers in hexagonal boron nitride (h-BN) are among the brightest emission centers known, yet the origins of these emission centers are not well understood. Here, using first-principles calculations in combination with the generating function method, we systematically elucidate the coupling of specific defects to the vibrational degrees of freedom. We show that the line shape of many defects exhibits strong coupling to high frequency phonon modes and that $C_{N}, C_{B}, C_{B}-C_{N}$ dimer, and $V_{B}$ can be associated with experimental line shapes. Our detailed theoretical study serves as a guide to identify optically active defects in h-BN that can suit specific applications in photonic-based quantum technologies, such as single photon emitters, hybrid spin-photon interfaces, or spin-mechanics interfaces.
\end{abstract}

DOI: 10.1103/PhysRevB.103.115421

\section{INTRODUCTION}

Hexagonal boron nitride (h-BN) is a wide-band-gap vander-Waals solid. In its exfoliated form, h-BN is a stable two-dimensional material that retains its wide band gap of about $6 \mathrm{eV}$. This large band gap supports a diversity of optically active defect centers, exhibiting a wide range of emission energies between 1.2 and $5.3 \mathrm{eV}$ [1,2]. Recently, it was shown that mono and few-layer h-BN can host room-temperature single-photon emitters (SPEs) [2], which sparked enormous interest in the field of photonic-based quantum technologies $[3,4]$. Follow-up experiments studied SPEs in h-BN in more detail [2,5-10] and, remarkably, such emitters were shown to exhibit Fourier transform-limited emission up to room temperature [11]. An understanding of the optical properties of SPEs in h-BN would enable selecting specific defect centers to serve as single photon sources [4], as hybrid spin-photon [12] or spin-mechanics interfaces $[13,14]$.

In order to take full advantage of these SPEs, it is paramount to identify the responsible defect structures and corresponding electronic transitions. However, so far no consensus has been reached concerning the origin of single-photon emission. While the spatial localization of the single-photon emission strongly suggests point defects to be the culprit, the assignment to a specific defect in h-BN is hampered by the range of zero-phonon lines (ZPLs) that are observed and the large number of potential defect candidates.

*erhart@chalmers.se

Published by the American Physical Society under the terms of the Creative Commons Attribution 4.0 International license. Further distribution of this work must maintain attribution to the author(s) and the published article's title, journal citation, and DOI. Funded by Bibsam.
For ZPLs between 1.6 and $2.3 \mathrm{eV}$, in most cases pronounced phonon sidebands (PSBs) at about $165 \mathrm{meV}$ below the ZPL are observed [2,5-9]. In some experiments, PSBs for some SPEs exhibit, however, a double-peak structure at around $160 \mathrm{meV}$ and 190-200 meV [9,10]. The similarity of the PSBs across measurements suggests the emission to be due to either multiple different defects with similar geometry or a single defect with variable excitation energy. In a recent experiment, the variable excitation energy was attributed to strain effects [10], but Stark shifts have been suggested as well [15]. It has also been proposed that there are two families of emitters around $2 \mathrm{eV}$ with different electronic structure and ZPLs of 1.88 and $2.14 \mathrm{eV}$, respectively, that can be distinguished not only based on their ZPL but also their quantum efficiency [16].

h-BN also exhibits luminescence in the ultraviolet [17], with a recent experiment [18] demonstrating single photon emission at $4.1 \mathrm{eV}$. The structure of this defect remains unknown but has been proposed to originate from carbon defects, although the emission intensity does not exhibit correlation with C impurity concentration [19]. The highest intensity peak of the PSB of the $4.1 \mathrm{eV}$ emitter has been suggested to originate from coupling to a $187-\mathrm{meV}$ local phonon mode [17] and coupling to the zone center longitudinal optical (LO) phonon mode at $200 \mathrm{meV}$ [20].

Recent theoretical studies focused on calculating the electronic structure of point defects in monolayer and bulk h-BN based on first-principle methods [21,22]. The ZPLs for rather many defects have been calculated within the accuracy permitted by current density-functional theory (DFT) methods based on hybrid functionals. Furthermore, the PSBs have been analyzed using phenomenological models [9,23] using a few selected phonon modes. To the best of our knowledge, a combined defect and PSBs study has, however, only been performed on the defects $\mathrm{N}_{\mathrm{B}}-\mathrm{V}_{\mathrm{N}}$ and $\mathrm{C}_{\mathrm{B}}-\mathrm{V}_{\mathrm{N}}$ [21,24]. In the latter studies it was concluded that the calculated emission spectra of $\mathrm{N}_{\mathrm{B}}-\mathrm{V}_{\mathrm{N}}$ do not agree with the measured line shapes 
but that $\mathrm{C}_{\mathrm{B}}-\mathrm{V}_{\mathrm{N}}$ might be a SPE. Besides these two defects, information about how specific defects couple to the vibrational degrees of freedom is scarce.

In the present work, we contribute to closing this knowledge gap concerning point defect-related emissions in h-BN by considering both charged and charge neutral transitions and the resulting emission line shape for a set of the most common intrinsic and extrinsic point defects. Importantly, we calculate the combined defect and PSB emission spectrum and, thus, can assess the vibrational fine structure. The resulting emission spectrum can be used as an experimentally accessible fingerprint to identify defect-related SPEs. This is possible since the vibrational fine structure of the emission spectrum due to an electronic transition on a point defect is highly sensitive to changes in the local distortion between initial and final state [25]. In the following, we consider vacancies $\left(\mathrm{V}_{\mathrm{N}}\right.$, $\left.\mathrm{V}_{\mathrm{B}}\right)$ and antisites $\left(\mathrm{N}_{\mathrm{B}}, \mathrm{B}_{\mathrm{N}}\right)$ as the most important intrinsic defects as well as carbon impurities $\left(\mathrm{C}_{\mathrm{B}}, \mathrm{C}_{\mathrm{N}}\right)$, vacancy-impurity complexes $\left(\mathrm{C}_{\mathrm{B}}-\mathrm{V}_{\mathrm{N}}, \mathrm{C}_{\mathrm{N}}-\mathrm{V}_{\mathrm{B}}\right)$ and one antisite-vacancy complex $\left(\mathrm{N}_{\mathrm{B}}-\mathrm{V}_{\mathrm{N}}\right)$. This selection covers most of the defects that have been proposed as SPEs in h-BN.

\section{METHODOLOGY}

\section{A. Defect formation energy} by

The formation energy of a defect in charge state $q$ is given

$$
\Delta E_{f}=E_{\text {defect }}-E_{\text {ideal }}-\sum \Delta N_{i} \mu_{i}+q\left(\varepsilon_{\mathrm{VBM}}+\Delta \mu_{e}\right),
$$

where $E_{\text {defect }}$ and $E_{\text {ideal }}$ are the total energies of the defective and ideal systems, respectively. $\Delta N_{i}$ denotes the change in the number of atoms of type $i$ between defective and ideal system, while $\varepsilon_{\mathrm{VBM}}$ and $\Delta \mu_{e}$ are the valence band maximum (VBM) position and the (relative) electron chemical potential, respectively. The chemical potentials $\mu_{i}$ of $\mathrm{B}$ and $\mathrm{N}$ are coupled to each other via

$$
\mu_{\mathrm{B}}+\mu_{\mathrm{N}}=-E_{\mathrm{BN}}
$$

where $E_{\mathrm{BN}}$ is the cohesive energy of h-BN [26]. Below, we consider the nitrogen-rich limit, where $\mu_{\mathrm{N}}=-\frac{1}{2} E_{\mathrm{N}_{2}}$ and the boron-rich limit, where $\mu_{\mathrm{B}}$ is taken as the negative cohesive energy of elemental $\alpha$-B (spacegroup $\mathrm{R} \overline{3} \mathrm{~m}$ ). The chemical potential of carbon is set to the one of graphene throughout. The charge transition level (CTL) between charge states $q$ and $q^{\prime}$ is the value of the electron chemical potential for which the formation energies of the defect in charge state $q$ and $q^{\prime}$ are equal. Throughout this study, CTLs are reported with respect to the VBM.

\section{B. Line shape of emission spectrum}

PSBs arise due to emission from the electronic excited state to the vibrationally excited electronic ground state. The structure of the emission spectrum can be computed from a knowledge of the phonon spectrum and the difference in the ionic configurations associated with excited and ground states $\Delta \boldsymbol{R}$. The extent of the lattice distortion can be measured by the magnitude of the mass weighted difference in ionic displacements

$$
\Delta Q=\sqrt{\sum_{a} m_{a} \Delta \boldsymbol{R}_{a} \cdot \Delta \boldsymbol{R}_{a}}
$$

where the sum runs over all atoms in the defect cell.

In this work, the line shape is computed using the generating function approach $[25,27,28]$. The central quantity in the generating function approach is the electron-phonon spectral function, which depends on the coupling between lattice displacement and vibrational degrees of freedom. The latter information is encoded in the so-called partial Huang-Rhys (HR) factor

$$
S_{k}=\frac{1}{2 \hbar} \omega_{k} Q_{k}^{2} .
$$

$Q_{k}$ is the projection of the lattice displacement on the normalized collective displacement described by phonon mode $k$ and given by [29]

$$
Q_{k}=\sum_{a} \sqrt{m_{a}}\left(\boldsymbol{n}_{k}^{a} \mid \Delta \boldsymbol{R}_{a}\right),
$$

where $a$ runs over all the atoms in the computational cell and $n_{k}$ is the normalized ionic displacement vector corresponding to phonon mode $k$.

The electron-phonon spectral function is then obtained by summation over all modes

$$
S(\omega)=\sum_{k} S_{k} \delta_{\omega, \omega_{k}} .
$$

It has dimensions of inverse energy in the same units as $\omega$. The integral over the electron-phonon spectral function gives the (total) HR factor of the transition. The electron-phonon spectral function is then transformed to the time domain to obtain $S(t)=\int d \omega S(\omega) \exp (-i \omega t)$ and the generating function $G(t)$ is obtained by exponentiation of $S(t)$

$$
G(t)=e^{S(t)}
$$

This expression is obtained in the low temperature and parallel mode approximation (see Sec. II C below). Fourier transformation of the generating function yields the line-shape function [29]

$$
A\left(\omega_{e g}-\omega\right)=\frac{1}{2 \pi} \int_{-\infty}^{\infty} d t G(t) e^{i \omega t-\kappa|t|}
$$

Here $\kappa$ is a broadening parameter that governs the width of the ZPL. It is necessary for numerical reasons and does not represent thermal broadening. It can, however, be tweaked to (artificially) broaden the spectrum and specifically the ZPL, which in measurements are subject to inhomogeneous and thermal broadening. The $\delta$ functions in Eq. (6) are approximated with normalized Gaussians, which is akin to the smearing that is adopted when computing electronic and phonon densities of states. Similarly, there is a correlation between the convergence with respect to smearing and the number of modes (i.e., the size of the supercell in the present case or the density of the Brillouin zone mesh when computing a density of states). Here, we use a smearing width of $6 \mathrm{meV}$ to broaden the Gaussians. 
Finally, the line-shape function is related to the luminescence intensity via

$$
L(\omega)=C \omega^{3} A(\omega),
$$

where $C$ is a normalization constant chosen such that $\int d \omega L(\omega)=1$.

The localization of a phonon mode can be measured using the inverse participation ratio (IPR) [29]

$$
\operatorname{IPR}_{k}=1 / \sum_{a}\left(\boldsymbol{n}_{k}^{a} \cdot \boldsymbol{n}_{k}^{a}\right)^{2}
$$

which can assume values between 1 and $N$, where $N$ is the number of atoms in the computational cell for which the phonon spectrum has been calculated. Smaller and larger values indicate more and less localized character.

\section{Limitations of the generating function method}

For reasons of computational complexity one usually makes several approximations when applying the generating function approach to predict the line shapes of specific defects. As note above (Sec. II B), here, we employ the lowtemperature and parallel mode approximations.

In the low-temperature approximation the emission is assumed to occur from the vibrational ground state of the excited electronic state. In addition, anharmonic contributions, which lead to, e.g., finite phonon lifetimes, are neglected.

In general, the normal modes of the initial $\vec{Q}_{i}$ and final configurations $\vec{Q}_{f}$ can be related via the Duschinsky transformation [30,31]

$$
\vec{Q}_{f}=\mathbf{J} \vec{Q}_{i}+\vec{K},
$$

where $\mathbf{J}$ and $\vec{K}$ represent a rotation and a translation, respectively. In the parallel mode approximation $\mathbf{J}$ is taken as the identity matrix. As a result, the evaluation of highdimensional integrals involving the ionic wave functions factorizes into products over one-dimensional integrals, dramatically reducing the computational cost. For systems with relatively few atoms (e.g., molecules) it is feasible to avoid the parallel mode approximation but it is often required for practical reasons in solids [31]. It can, however, lead to errors in particular for transitions in which the ground and excited state configurations exhibit different symmetries.

Furthermore, we note that in many measurements the photoluminescence is measured over a finite region containing an ensemble of defects with variations in the local environment (e.g., local strain), leading to inhomogeneous broadening, which is not captured here.

\section{Computational details}

All DFT calculations were carried out using plane-wave basis sets [32] and the projector augmented wave method $[33,34]$ as implemented in the Vienna ab initio simulation package [35] (version 5.4.4). Exchange-correlation contributions were obtained using the semilocal functional of Perdew, Burke, and Ernzerhof (PBE) [36] and the hybrid HSE06 functional [37] using both the standard value for the mixing parameter $\alpha=0.25$ as well as a value of $\alpha=0.60$ tuned to reproduce the band gap as detailed below (Sec. III A). A plane wave basis set with a cutoff energy of $550 \mathrm{eV}$ was employed to represent the electronic wave functions. Geometry optimization was performed for all systems, during which the atomic positions were allowed to relax until all forces were less than $20 \mathrm{meV} / \AA$.

Brillouin zone sampling was performed using a grid of $21 \times 21 \times 1$ for the primitive hexagonal (two-atom) unit cell. Defect calculations were carried out using a $8 \times 8 \times 1$ supercell with $19.88 \AA$ vacuum while the Brillouin zone was sampled using the $\Gamma$-point only.

The ZPLs arising from transitions between defect-induced levels and band states were computed using the HSE06 hybrid functional using a mixing parameter of $\alpha=0.6$ to correct for the band-gap error of semilocal functionals (see Sec. III A for the motivation of this choice of mixing parameter). For charged defect cells, a correction of $q^{2} 0.5 \mathrm{eV}$ was added to the total energy to account for periodic image charge effects and potential offsets $[38,39]$. This approach yields CTLs that agree well (within $30 \mathrm{meV}$ ) with the values presented in the erratum of Ref. [38].

Charge neutral excitations were modelled using the $\triangle \mathrm{SCF}$ method, in which the electronic occupations are constrained. The description of electronic states that are localized at the level of semilocal exchange-correlation functionals such as PBE is commonly only weakly affected by the addition of exact exchange. (For illustration, PBE and standard HSE06 ( $\alpha=$ 0.25 ) yield $\mathrm{ZPLs}$ for $\mathrm{V}_{\mathrm{B}}^{-}$of 1.71 and $1.84 \mathrm{eV}$, respectively.) Since in the present case the convergence of excited state calculations with hybrid functionals is both very tedious and computationally demanding, all charge neutral defect-defect transitions were treated at the semilocal density functional theory (DFT) level (PBE).

Vibrational spectra were obtained at the semilocal DFT level (PBE) using the PHONOPY package [40]. In the computation of the electron phonon spectral function $S(\omega)$, the Kronecker $\delta$ in Eq. (6) was approximated using normalized Gaussians with a smearing of $6 \mathrm{meV}$. The integration over the time domain in the Fourier transform to obtain $A(\omega)$ was performed over 2 ps with a time spacing of $1 \mathrm{fs}$. Convergence of the spectral distribution function is demonstrated in the Supplemental Material [41].

\section{RESULTS}

\section{A. Pristine h-BN}

Without taking into account zero-point effects, the PBE functional yields a lattice parameter of $2.51 \AA$ for $\mathrm{h}$-BN while one obtains $2.49 \AA$ with HSE06 using $\alpha=0.25$. These values compare well with the experimental lattice parameter for bulk $\mathrm{BN}$ of $2.51 \AA$ at $10 \mathrm{~K}$ [42]. For consistency, all calculations below, including those based on the standard HSE06 functional (mixing parameter $\alpha=0.25$ ), were carried out using a lattice constant of $2.51 \AA$.

The calculated electronic (or single-particle) band gap, on the other hand, measures 4.67 and $5.71 \mathrm{eV}$ with PBE and standard HSE06 $(\alpha=0.25)$, respectively, in line with other theoretical studies [43]. The experimentally measured optical band gap is $6.1 \mathrm{eV}$ and $6.0 \mathrm{eV}$ for monolayer h-BN [44] and bulk BN [45], respectively. While these numbers appear rather 


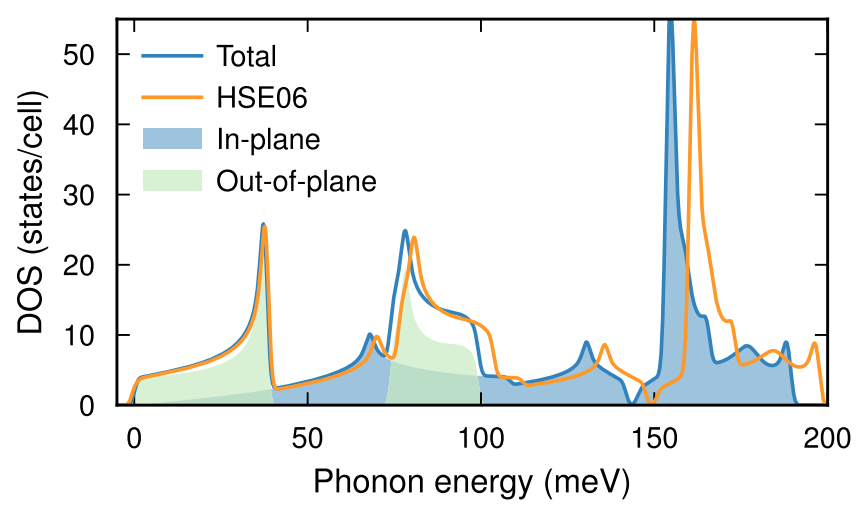

FIG. 1. Vibrational spectrum of pristine h-BN with Cartesian projection of the normal modes as computed with PBE and HSE06 $(\alpha=0.25$ and total density of states only; computed using a $8 \times 8 \times 1$ supercell).

close to the value obtained using standard HSE ( $\alpha=0.25)$, a sound comparison requires accounting for the exciton binding energy, which is very large both in bulk [46] and monolayer h-BN [43], as well as the effect of zero-point motion via electron-phonon coupling [47]. It is therefore more instructive to compare the DFT values with the results from $G_{0} W_{0}$ calculations [43], which provide a value of $7.1 \mathrm{eV}$, corresponding to the single-particle band gap in the absence of both exciton formation and zero-point motion. To reach this value using the HSE06 functional one requires a mixing parameter of $\alpha=0.60$, yielding a band gap of $7.2 \mathrm{eV}$. Below, we therefore use HSE06 with a mixing parameter of $\alpha=0.60$ to compute defect formation energies and ZPLs derived thereof.

Before considering the vibrational spectra of defects, a closer inspection of the vibrational spectrum of pristine h-BN is instructive. The vibrational spectra from PBE and HSE06 are very similar, with the latter yielding a slightly stiffer response overall (Fig. 1) [48]. Given the two-dimensional (2D) character of h-BN, the phonon density of states (DOS) can be decomposed into an in-plane and out-of-plane part. In part due to the quadratic dispersion inherent to 2D materials [49], the lower frequency part of the spectrum is dominated by out-of-plane modes. The out-of-plane partial DOS features two pronounced bands ranging from 0 to about $40 \mathrm{meV}$ and from approximately 65 to $100 \mathrm{meV}$ (values from PBE), respectively, with two pronounced peaks at 40 and $80 \mathrm{meV}$. The in-plane partial DOS covers the entire frequency range spanning up to $187 \mathrm{meV}$ in good agreement with other firstprinciple studies [50,51]. The most notable feature is an asymmetric peak at $154 \mathrm{meV}$.

\section{B. Defect energetics}

From the outset we considered vacancies $\left(\mathrm{V}_{\mathrm{N}}, \mathrm{V}_{\mathrm{B}}\right)$ and antisites $\left(\mathrm{N}_{\mathrm{B}}, \mathrm{B}_{\mathrm{N}}\right)$ as possibly relevant intrinsic defects as well as carbon impurities $\left(C_{B}, C_{N}, C_{B}-C_{N}\right)$, vacancy-impurity complexes $\left(\mathrm{C}_{\mathrm{B}}-\mathrm{V}_{\mathrm{N}}, \mathrm{C}_{\mathrm{N}^{-}} \mathrm{V}_{\mathrm{B}}\right)$ and one antisite-vacancy complex $\left(\mathrm{N}_{\mathrm{B}}-\mathrm{V}_{\mathrm{N}}\right)$, see Fig. 2(a) for atomic structures. To determine the energetically most favorable defects and defect configurations along with pertinent charge states we computed the defect formation energies under both $\mathrm{B}$ and $\mathrm{N}$-rich conditions [Fig. 2(b)]. Where comparable the results are consistent with previous work on monolayer h-BN [38]. Under N-rich conditions $\mathrm{N}_{\mathrm{B}}$ (donor with $q=+1,0$ ) and $\mathrm{V}_{\mathrm{B}}$ (acceptor with $q=0,-1)$ are the most favorable intrinsic defects, whereas under B-rich conditions $\mathrm{B}_{\mathrm{N}}$ (acceptor, $q=0,-1$ ) and $\mathrm{V}_{\mathrm{N}}$ (ambipolar with $q=+1,0,-1$ ) have the lowest formation energies. With regard to extrinsic defects, we find $\mathrm{C}_{\mathrm{B}}(q=$ $+1,0), \mathrm{C}_{\mathrm{N}}(q=0,-1)$, and $\mathrm{C}_{\mathrm{B}}-\mathrm{C}_{\mathrm{N}}(q=0,-1)$ to be lowest energy defects under both $\mathrm{B}$ and $\mathrm{N}$-rich conditions. The defect complexes involving substitutional impurities and vacancies, specifically $C_{B}-V_{N}$ and $N_{B}-V_{N}$, have high formation energies but are nonetheless included in the analysis below since they have been discussed as potential SPEs before [2,21,52].

\section{Transition types}

In the following, we consider two types of transitions: (i) localized-to-delocalized (LD) transitions involve a localized defect level and a (delocalized) band edge state; (ii) localizedto-localized (LL) transitions involve two defect levels both of which reside inside the band gap when they are occupied.

To illustrate the features and emphasize similarities and differences between these transition types, it is instructive to (a)

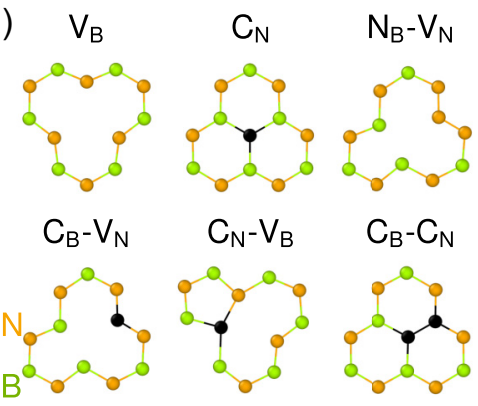

(b)

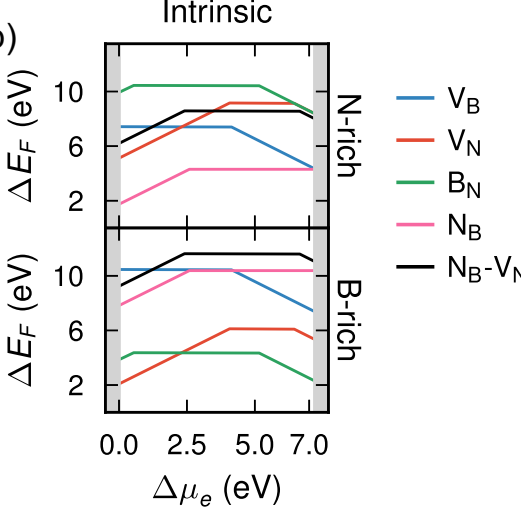

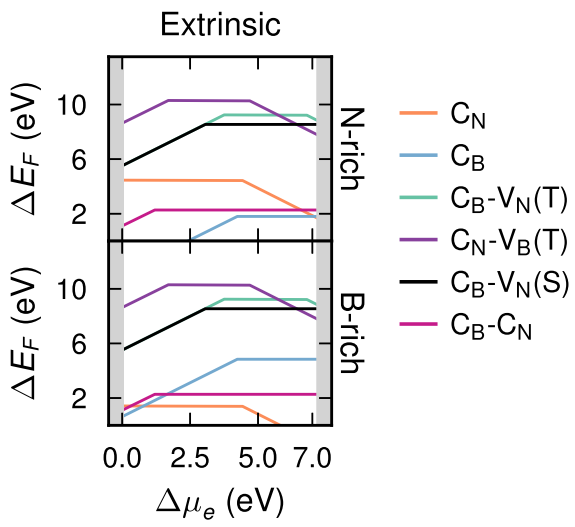

FIG. 2. (a) Structures of a selection of defects in h-BN. Boron, nitrogen, and carbon atoms are shown in green, orange, and black, respectively. (b) Formation energies of intrinsic (left) and extrinsic (right) defects in the N-rich (top) and B-rich (bottom) limits. S and T indicate singlet and triplet configurations, respectively. 


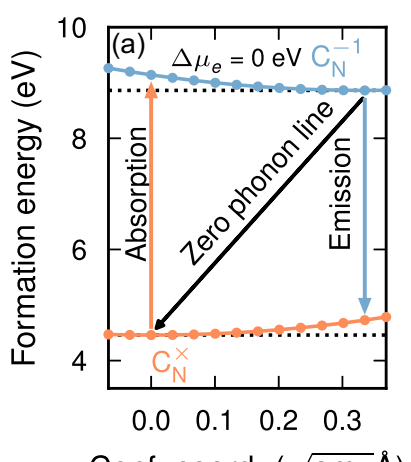

Conf. coord. $(\sqrt{\mathrm{amu}} \AA)$
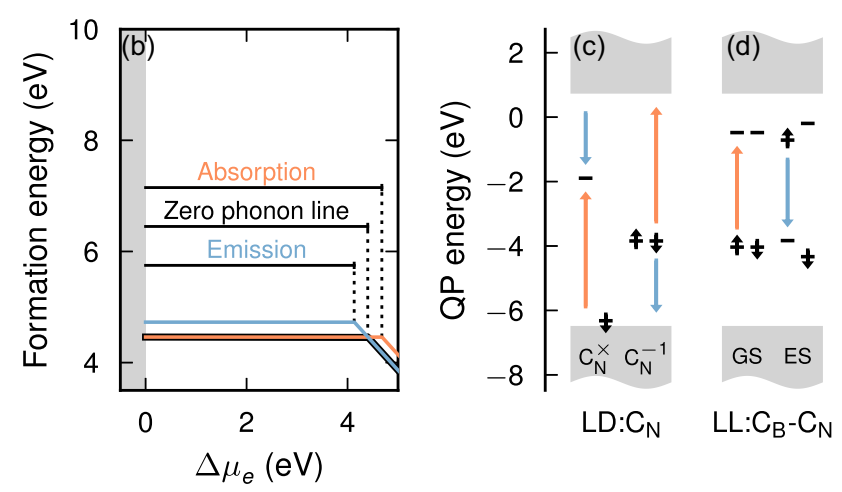

FIG. 3. Relation between (a) transitions in the one-dimensional configuration coordinate (CC) diagram and (b) the corresponding formation energy diagram illustrated for the case of recombination with valence band (VB) charge carriers $\left(\Delta \mu_{e}=0 \mathrm{eV}\right)$ in $\mathrm{C}_{\mathrm{N}}$. The CC diagram is constructed based on PBE values and shifted to the HSE06 ( $\alpha=0.60)$ ZPLs. The difference between localized-localized (LL) and localizeddelocalized (LD) transitions is schematically shown in (c) and (d), where GS and ES stand for ground state and excited state, respectively, and the arrows indicate electrons in spin-up and spin-down states, respectively. In (e) the one-dimensional CC diagram is shown for the LL transition on $\mathrm{C}_{\mathrm{B}}-\mathrm{C}_{\mathrm{N}}$. The $1 \mathrm{D}$ CC diagram has been computed with PBE.

recall the relations between configuration coordinate diagram, defect formation energies, CTLs, and defect levels.

In the case of LD transitions, illustrated here by $\mathrm{C}_{\mathrm{N}}$, the absorption (emission) energy corresponds to the $0 /-1$ CTL obtained when the atomic configuration is constrained to the equilibrium $\mathrm{C}_{\mathrm{N}}^{0}\left(\mathrm{C}_{\mathrm{N}}^{-}\right)$geometry [Fig. 3(a)]. The ZPL then corresponds to the $\mathrm{CTL}$ obtained for the equilibrium geometries in either charge state [Fig. 3(b)]. If the electron chemical potential $\Delta \mu_{e}$ coincides with the VBM, emission occurs by capturing a hole from the VB edge, depleting the defect level associated with $\mathrm{C}_{\mathrm{N}}$ [Fig. 3(c)]. If the electron chemical potential resides at the conduction band minimum (CBM) the initial and final states are inverted. Since a delocalized band state is involved in this transition type, a proper description of the position of the band edges is crucial.

To illustrate LL transitions, we consider here the $C_{B}-C_{N}$ defect, which presents a particular simple two-level system. Emission occurs from the excited state $C_{B}-C_{N}^{0, *}$, in which the highest occupied defect state resides above the lowest unoccupied level, to the ground state $\mathrm{C}_{\mathrm{B}}-\mathrm{C}_{\mathrm{N}}^{0}$ [Fig. 3(d)]. The difference in character of the highest occupied defect level in ground and excited states implies a difference in local potential that gives rise to a considerable lattice relaxation after emission [Fig. 3(e)] that underlies the Stokes shift.

The possible LD and LL transitions involving the defects considered here are compiled in Table I.

\section{Transitions on intrinsic defects}

Next we turn to a survey of the transition energies and subsequently an assessment of the line shapes of intrinsic defects. The prominent high-frequency PSB of emitters in the 1.6- to $2.3-\mathrm{eV}$ range and at $4.1 \mathrm{eV}$ indicates an effective phonon frequency of $\omega_{\text {eff }} \sim 100 \mathrm{meV}$. The effective frequency is coupled to the HR factor $S$ and the lattice distortion connecting the initial and final configurations $\Delta Q$ according to

$$
S=\omega_{\text {eff }} \Delta Q^{2} / 2 \hbar \text {. }
$$

As experimentally measured HR factors fall in the range of $S=1$ to 2 , the lattice distortion of potential emitters must therefore be relatively small. For example, a transition coupled to an effective mode with a frequency of $100 \mathrm{meV}$ must have $\Delta Q<0.41$ in order to have an HR factor below two.

Most transitions on intrinsic defects exhibit, however, rather large lattice distortions with $\Delta Q$ well above $1 \sqrt{\mathrm{amu}} \AA$ (see $\Delta Q$ in Table I) that disqualify them as possible narrow band emitters. These defects often exhibit geometries in which one or several atoms are located outside of the BN plane, which can be related to the large structural differences between different electronic states. Transitions involving $\mathrm{V}_{\mathrm{N}}$ and $\mathrm{N}_{\mathrm{B}}$ are therefore not considered further.

$\mathrm{B}_{\mathrm{N}}$ can host a LL transition with a ZPL of $1.14 \mathrm{eV}$ according to PBE with a relatively small $\Delta Q$. As the transition is rather far from the spectral range of interest here $(>1.6 \mathrm{eV})$, transitions on $\mathrm{B}_{\mathrm{N}}$ are not considered further either.

$\mathrm{N}_{\mathrm{B}}-\mathrm{V}_{\mathrm{N}}$ can be ruled out as well based on the large value of $\Delta Q$. The one remaining intrinsic defect $\mathrm{V}_{\mathrm{B}}$ is considered in detail in the following section.

\section{Boron vacancy $\left(\mathrm{V}_{B}\right)$}

The symmetry of $\mathrm{V}_{\mathrm{B}}^{0}$ is $C_{2 v}$, while the symmetry of $\mathrm{V}_{\mathrm{B}}^{-}$is $D_{3 h}$. The $0 /-1$ CTL resides $4.12 \mathrm{eV}$ above the VBM, which is much larger than an earlier theoretical value of $2.4 \mathrm{eV}$ [53]. The latter though was obtained using a much smaller mixing parameter of $\alpha=0.32$, which as discussed in Sec. III A yields a misleading agreement with the experimentally measured optical band gap. The large difference is caused by a change in the spin state of $\mathrm{V}_{\mathrm{B}}^{0}$ with increasing mixing parameter ( $\alpha=0.25: S=3 / 2 ; \alpha=0.60: S=1 / 2)$ that pushes the CTL to higher energies with respect to the VBM than the corresponding rigid band shift.

At the standard HSE06 level $(\alpha=0.25), \mathrm{V}_{\mathrm{B}}^{-}$induces nine occupied in-gap states and two unoccupied in-gap states. In the spin-up channel, in which the optical transitions presumably occur, there are five occupied in-gap states and two degenerate unoccupied states. There are two possible LD transitions on $\mathrm{V}_{\mathrm{B}}$, namely a $\mathrm{VB}$ hole capture transition involving $\mathrm{V}_{\mathrm{B}}^{-}$and a conduction band $(\mathrm{CB})$ electron capture by $\mathrm{V}_{\mathrm{B}}^{0}$ with 
TABLE I. Transition energies and structural distortions associated with different transitions computed with PBE for localized-localized transitions and HSE06 for localized-delocalized transitions. HSE06 values were obtained by using HSE06 with $\alpha=0.6$ on the standard HSE06 $(\alpha=0.25)$ ionic configuration. For localized-delocalized transitions both the ZPL corresponding to the transition involving the VB edge (left column; identical to the charge transition level) and the transition involving the CB edge (right column) are given [see Fig. 3(c)]. Arrows indicate the direction of a transition. Singlet and triplet states are marked by $(\mathrm{S})$ and $(\mathrm{T})$, respectively. Localized-delocalized transitions can proceed in either direction, whereas localized-localized transitions always commence from the excited state, the latter being marked by asterisks. The "Modes" column identifies which phonon spectrum was used for the computation of the line-shape function. ZPL: zero-phonon line; $\Delta Q$ : magnitude of the lattice distortion between the two defect configurations involved in the transition; HR: Huang-Rhys factor.

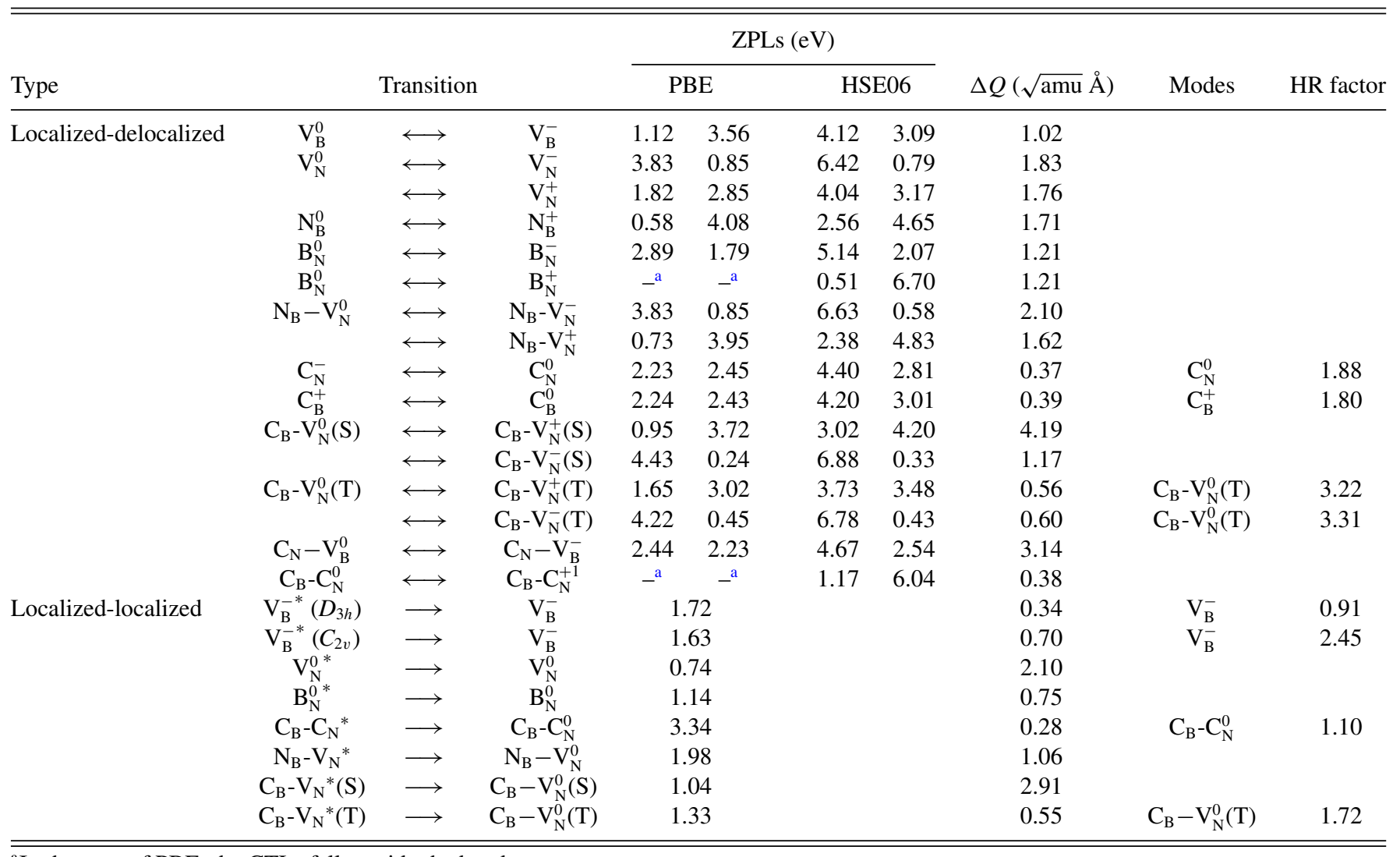

${ }^{\mathrm{a}}$ In the case of PBE, the CTLs fall outside the band gap.

ZPLs of 4.12 and $3.09 \mathrm{eV}$, respectively. The lattice distortion between $\mathrm{V}_{\mathrm{B}}^{0}$ and $\mathrm{V}_{\mathrm{B}}^{-}$is quite large at $1.0 \sqrt{\mathrm{amu}} \AA$.

Furthermore, there are five possible LL transitions involving $\mathrm{V}_{\mathrm{B}}^{-}$. Here, we consider only the highest occupied molecular orbital (HOMO)/lowest unoccupied molecular orbital (LUMO) transition in both the $D_{3 h}$ and $C_{2 v}$ symmetries of the excited state with ZPLs of 1.72 and $1.63 \mathrm{eV}$, respectively. The difference of $\sim 90 \mathrm{meV}$ between the considered ionic configurations of the excited state suggests that $C_{2 v}$ should be the equilibrium configuration. Below we, however, consider the line shapes of both the configurations with $D_{3 h}$ and $C_{2 v}$ symmetry, due to the small energy difference and the possibility that semilocal DFT provides an incorrect description of the equilibrium ionic configuration of the excited state.

First, the case where the initial state symmetry is $C_{2 v}$ is considered with a total HR factor of 2.45. In this case there is a strong coupling of the electronic transition to a single mode at $26 \mathrm{meV}$ with a partial HR factor of $0.66(27 \%$ of the total HR factor) (the spectral function is shown in the Supplemental Material [41], Fig. S9). The IPR of this mode is 92, suggesting that it is likely a delocalized mode (the maximal value of the IPR that can be reached in this supercell is 128). At energies around $40 \mathrm{meV}$, there are three modes with partial HR factors of 0.11 to 0.29 with IPRs of 40 to 50 . In the high-frequency end, there is a coupling to a $162 \mathrm{meV}$ mode with a partial HR factor of 0.05 ( $2 \%$ of the total HR factor). The resulting normalized emission line shape is shown in Fig. 8, where the spectrum has been broadened by tuning the damping parameter $(\kappa)$ in Eq. (7) (see Fig. S11 for an illustration of how the spectral distribution function changes with increasing $\kappa$ in the case of $\mathrm{C}_{\mathrm{N}}$ ).

Next, the emission line shape of the transition from the $D_{3 h}$ symmetric initial state is considered [Fig. 4(a)], which has a much smaller total HR of 0.91. The spectral function [Fig. 4(b)] has two prominent peaks at 40 and $162 \mathrm{meV}$. The 40-meV peak results from the coupling to a single phonon mode and the coupling to other phonons with energy in the vicinity of $40 \mathrm{meV}$ is very weak. The IPR of the $40 \mathrm{meV}$ mode is 50 and the partial HR of this mode is 0.34 (37\% of total HR). The phonon eigenvector for the $40-\mathrm{meV}$ and the $162-\mathrm{meV}$ mode is shown in Fig. 4(b). The IPR of the 162-meV mode is 26 and the partial HR factor is 0.07 . While this value is slightly larger than the corresponding value in the emission from the $C_{2 v}$ initial state, the relative contribution is much larger at $8 \%$ of the total HR factor. Therefore, the peak at $162 \mathrm{meV}$ in the emission line shape is much more pronounced 

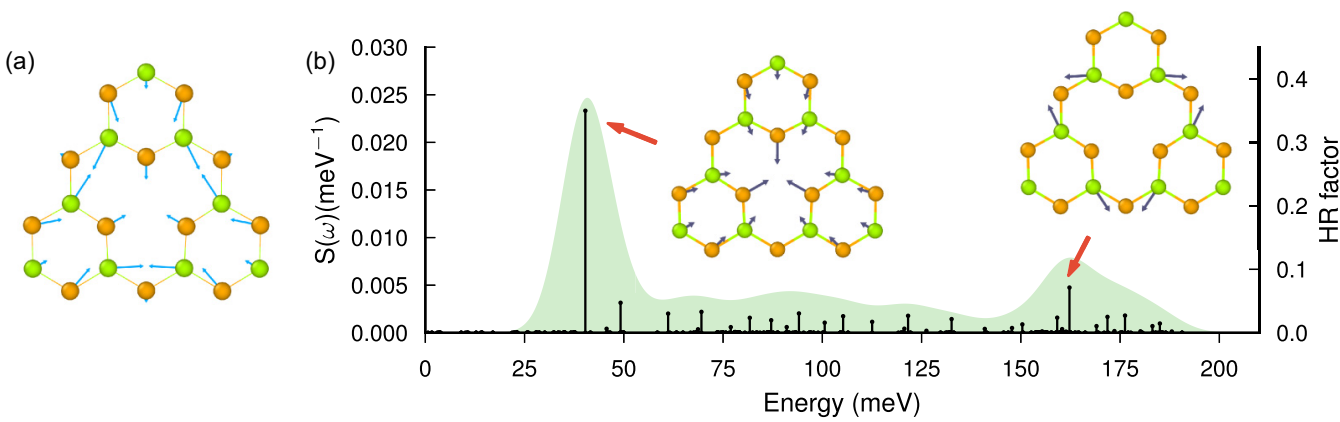

FIG. 4. (a) Ionic displacement in the $D_{3 h} \mathrm{HOMO} / \mathrm{LUMO}$ transition on $\mathrm{V}_{\mathrm{B}}^{-}$. (b) Spectral function and ionic displacements due to the dominant phonon modes.

in the case of emission from the $D_{3 h}$ state. The normalized emission line shape of the emission from the $D_{3 h}$ is shown in Fig. 8.

\section{E. Transitions on extrinsic defects}

Compared with intrinsic defects among which only $V_{B}$ is a viable candidate for single-photon emission, extrinsic defects involving carbon feature a multitude of suitable electronic transitions (Table I). Transitions involving $\mathrm{C}_{B}-\mathrm{V}_{\mathrm{N}}(\mathrm{S})$ and $\mathrm{C}_{\mathrm{N}}-\mathrm{V}_{\mathrm{B}}$ exhibit large lattice distortions and are therefore excluded from further analysis as argued above. In the following we therefore focus on $C_{N}, C_{B}, C_{B}-V_{N}(T)$, as well as $\mathrm{C}_{\mathrm{B}}-\mathrm{C}_{\mathrm{N}}$ dimers.

\section{Carbon-on-nitrogen $\left(\mathrm{C}_{N}\right)$}

The ideal h-BN structure is only modified slightly with the inclusion of a $\mathrm{C}_{\mathrm{N}}^{0}$. $\mathrm{C}_{\mathrm{N}}^{0}$ in $D_{3 h}$ symmetry exhibits a single unoccupied defect level within the band gap, indicating that only LD transitions are possible [Fig. 3(c)]. The 0/ - 1 CTL (ZPL for hole capture on $\mathrm{C}_{\mathrm{N}}^{-}$) resides at $4.40 \mathrm{eV}$. This is higher energy than earlier calculations for bulk h-BN that obtained a value of $2.84 \mathrm{eV}$ [22]. This can again be primarily attributed to the smaller mixing parameter of $\alpha=0.31$ (see Sec. III A and Sec. III D 1). It is significantly larger than measurements that located the $\mathrm{C}_{\mathrm{N}}$ acceptor at $2.3 \mathrm{eV}$ above the VBM [54]. The other possible transition is via $\mathrm{CB}$ electron capture on $\mathrm{C}_{\mathrm{N}}^{0}$, which has a ZPL of $2.81 \mathrm{eV}$. The lattice distortion associated with these transitions is only $0.37 \sqrt{\mathrm{amu}} \AA$.

The transitions on $\mathrm{C}_{\mathrm{N}}$ couple strongly to high frequency phonon modes, with the spectral function (Fig. S9 [41]) exhibiting distinct peaks at 158 and $185 \mathrm{meV}$. The $158-\mathrm{meV}$ peak consists of a single phonon mode that has a HR factor of 0.23 (12.4\% of the total HR factor of 1.88). The IPR of the $158-\mathrm{meV}$ mode is 27 . The $185-\mathrm{meV}$ peak on the other hand consists of several modes between 182 and $187 \mathrm{meV}$. The largest contribution to the spectral function comes from one mode at $182 \mathrm{meV}$ with a HR factor of 0.19 and an IPR of 73, and two modes at $187 \mathrm{meV}$ with HR factors of 0.19 and 0.22 . The IPR of the $187-\mathrm{meV}$ modes are 75 and 73 , respectively. The normalized emission line shape is shown in Fig. 9(a).

In order to elucidate the structural origin of the PSB, the ionic displacement due to the electronic transition can be overlaid with the phonon displacement vector for the highest frequency mode at $187 \mathrm{meV}$ (Fig. 5). The coupling between lattice and electronic transition in $\mathrm{C}_{\mathrm{N}}$ is dominated by the displacement of $\mathrm{B}$ atoms. The $\mathrm{B}$ ions closest to $\mathrm{C}_{\mathrm{N}}^{-}$experience the largest displacement on transition to $\mathrm{C}_{\mathrm{N}}^{0}$. However, these ions are not displaced in the phonon displacement vector so the contribution to the partial HR is essentially zero. The six B atoms in the next shell do not displace as much but contribute much more to the partial HR factor due to a much larger overlap with the phonon eigenvector. Finally, the B atoms in the third neighbor shell contribute the most to the partial HR factor.

The vibrational coupling can also be approximated by the 1D CC diagram in Fig. 3(a). We find that the effective frequencies $\omega_{\text {eff }}$ determined from the potential energy surface are 141 and $146 \mathrm{meV}$ for the ground state and excited state, respectively. These frequencies translate into HR factors [Eq. (11)] of 1.89 for the ground state and 1.95 for the excited state (1.88 with the generating function method). The effective frequencies are determined by the coefficient $a$ in the fitted polynomial $a Q^{2}+b Q^{3}$, from which we find $a / b>1000$ suggesting that the harmonic approximation is sound.

\section{Carbon-on-boron $\left(\mathrm{C}_{B}\right)$}

The possible charge transitions on $C_{B}$ have ZPLs of 4.20 and $3.01 \mathrm{eV}$, and the structural distortion associated with these

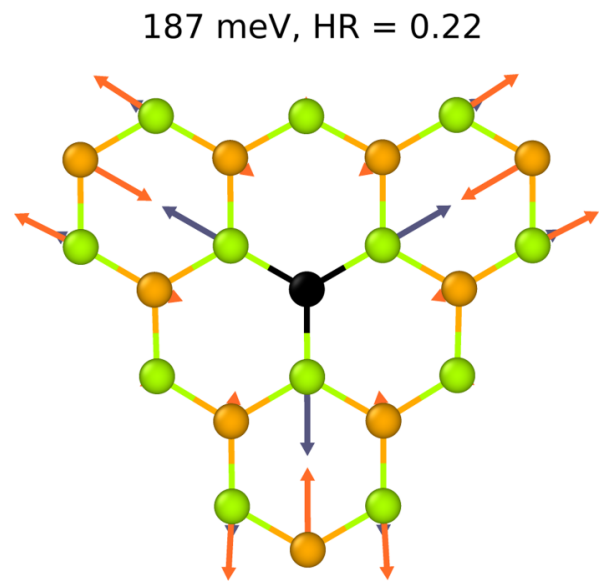

FIG. 5. Phonon normal mode (red arrows) and transition displacements (dark arrows) for the $187-\mathrm{meV}$ mode coupling in the charged transition on $\mathrm{C}_{\mathrm{N}}$. The phonon normal mode is amplified by a factor of 15 and the transition displacements are amplified by a factor of 30 . 


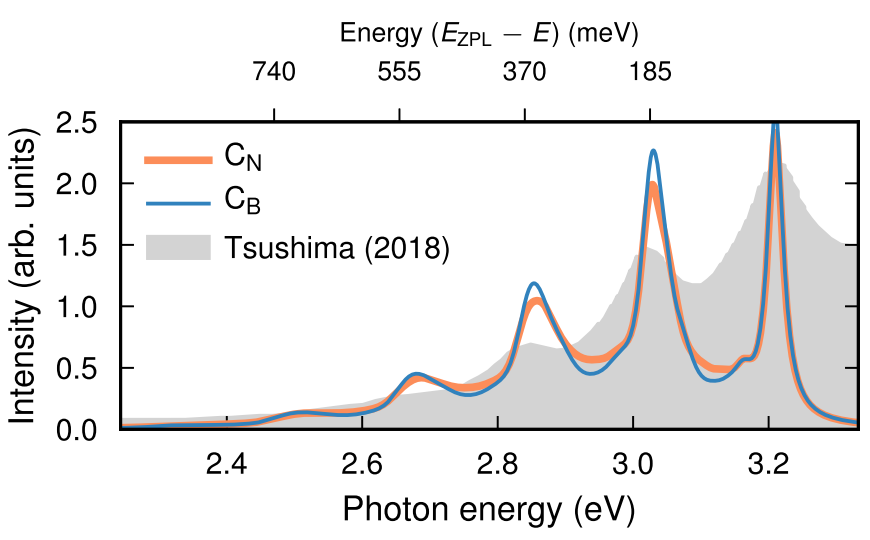

FIG. 6. Emission line shape of $C_{N}$ and $C_{B}$ defects compared with experimental results from Ref. [19]. For the calculated spectra ZPLs has been shifted to the experimental value of $3.22 \mathrm{eV}$. The experimental spectrum was measured at room temperature. The width of the ZPL has been tuned by setting $\kappa$ in Eq. (7) to $16 \mathrm{meV}$.

transitions is $0.34 \sqrt{\mathrm{amu}} \AA$. While the phonon spectrum for the $\mathrm{C}_{\mathrm{B}}^{0}$ defect contains imaginary modes, we were unable to find lower energy structure by eigenmode following. We attribute this finding to the very small energy difference associated with a displacement of the $\mathrm{C}_{\mathrm{B}}$ atom perpendicular to the h-BN plane (see Fig. S1 of the Supplemental Material [41]). The HR factor is 1.80 and there is a well-defined peak in the PSBs at $185 \mathrm{meV}$, with additional phonon replicas at higher energies. We note that the ZPLs and the spectral distribution function for $C_{B}$ are very similar to the ones in $C_{N}$ (Fig. 6 and S8 [41]), which would make it very difficult to distinguish between $C_{B}$ and $C_{N}$ in a photoluminescence experiment.

\section{Carbon-on-boron-nitrogen vacancy complex $\left(\mathrm{C}_{B}-\mathrm{V}_{N}\right)$}

The $C_{B}-V_{N}$ defect is found to have a singlet ground state in agreement with previous studies $[52,55]$ but as other studies have already pointed out a triplet electron configuration is also possible [21,56]. For the $C_{B}-V_{N}$ we considered three different structures: (i) the triplet planar configuration (T), (ii) the singlet planar configuration (S-planar), and (iii) the singlet structure in which the $\mathrm{C}$ atom is displaced out-of-plane (S).

The singlet planar (S-planar) structure is dynamically unstable confirming previous reports [57]. The triplet structure (T), on the other hand, is dynamically stable but about $0.2 \mathrm{eV}$ higher in energy than the S-planar configuration with PBE (compare Fig. S4 [41]). Finally, the singlet out-of-plane structure, which is $0.53 \mathrm{eV}$ lower in energy than the S-planar configuration, is both thermodynamically and dynamically stable and thus should be the equilibrium configuration of the $\mathrm{C}_{\mathrm{B}}-\mathrm{V}_{\mathrm{N}}$ defect. (A careful comparison of singlet and triplet configurations, including an assessment of the role of the exchange-correlation functional can be found in the Supplemental Material [41].)

While the singlet state is by far the most stable configuration, it can be ruled out as a SPE source due to the large value of $\Delta Q=2.91 \sqrt{\mathrm{amu}} \AA$. The LL transition in the triplet state, on the other hand, exhibits a much smaller lattice distortion. The ZPL, located at $1.33 \mathrm{eV}$, has a large intensity and there are multiple peaks in the PSB, occurring at energies between 28 and $157 \mathrm{meV}$ (Fig. S8 [41]). The high-energy peak is barely distinguishable from the spectral distribution function. The $\mathrm{HR}$ factor for this transition is 1.72 , in good agreement with previous studies on the line shape of $C_{B}-V_{N}$ in the triplet state [21]. The spectral distribution function for charged transitions on $\mathrm{C}_{\mathrm{B}}-\mathrm{V}_{\mathrm{N}}(\mathrm{T})$ exhibit a pronounced intensity on the ZPL (see Fig. S8 [41]). However, the PSB is wide and does not exhibit a shape that can be associated with experimental line shapes. The HR factor for these transitions are 3.2 and 3.3.

\section{Carbon-carbon dimer}

The $C_{B}-C_{N}$ dimer consists of $C_{N}$ and $C_{B}$ defects located on neighboring lattice sites [Fig. 2(a)] and has been suggested to form at high C-doping levels [54]. The C-C bond is significantly shorter than the corresponding pristine B-N distance, and a mode with higher frequency than any pristine $\mathrm{h}-\mathrm{BN}$ mode is present in the vibrational spectrum of the $C_{B}-C_{N}$ dimer at $195 \mathrm{meV}$.

The neutral charge state of the $\mathrm{C}_{\mathrm{B}}-\mathrm{C}_{\mathrm{N}}$ dimer is thermodynamically stable for Fermi levels above $1.17 \mathrm{eV}$ making charged transitions in either the $4.1 \mathrm{eV}$ or $1.6-$ to $2.3-\mathrm{eV}$ region impossible [Fig. 2(b)]. There are four in-gap single particle levels, two of which are occupied and two unoccupied, making a LL transition possible [Fig. 3(d)]. The emission energy for the charged $\mathrm{LL}$ transition on the $\mathrm{C}_{\mathrm{B}}-\mathrm{C}_{\mathrm{N}}$ dimer is $3.34 \mathrm{eV}$ and dipole allowed [Fig. 7(b)] while the lattice distortion between the ground and excited state equilibrium configurations is $0.28 \sqrt{\mathrm{amu}} \AA$.

The coupling of the charge neutral emission to the vibrational degrees of freedom has been analyzed with both the 1D CC diagram [Fig. 3(e)] and the generating function method. From the 1D CC we find that the effective frequencies are 110 and $127 \mathrm{meV}$ for the ground and excited state with HR factors based on the effective frequencies of 1.06 and 1.23 , respectively. In comparison to the $1 \mathrm{D} C \mathrm{C}$ for $\mathrm{C}_{\mathrm{N}}$, however, the third-order coefficient carries a much larger relative weight. The ratio between the second and third-order coefficients is below 300 in both cases suggesting that anharmonic effects are not completely negligible.

The spectral function $S(\omega)$ features a significant peak corresponding to coupling to the $195-\mathrm{meV}$ mode (Fig. S9 [41]), which correlates with a partial HR factor of 0.44 to be compared with the total HR factor of 1.10 as computed via the spectral function. The $195-\mathrm{meV}$ mode is localized, as indicated by the small value of the IPR of 4.5 , and corresponds to a stretching of the C-C bond. The computed HR factors of $1.10,1.06$, or 1.23 , depending on method of calculation, agree well with the measured HR factor for the 4.1-eV luminescence of 1.3 [17].

The computed normalized line shape compares very well with experimentally measured line shapes [18] for the 4.1-eV emission [Fig. 9(b)], including both the positions of the features in the PSBs and the relative intensities between the first and second peaks. We note that the results shown are for natural carbon, i.e., ${ }^{12} \mathrm{C}$. For ${ }^{13} \mathrm{C}$ the frequency of the dominating mode at $195 \mathrm{meV}$ is reduced to $191 \mathrm{meV}$.

\section{Dissociation of carbon-carbon dimer $\left(\mathrm{C}_{N}-\mathrm{C}_{B}\right)$}

Next we consider the effect of spatial separation on the $\mathrm{C}_{\mathrm{N}^{-}}$ $\mathrm{C}_{\mathrm{B}}$ defect, while the $\mathrm{C}-\mathrm{C}$ distance is varied between $2.90 \AA$ 

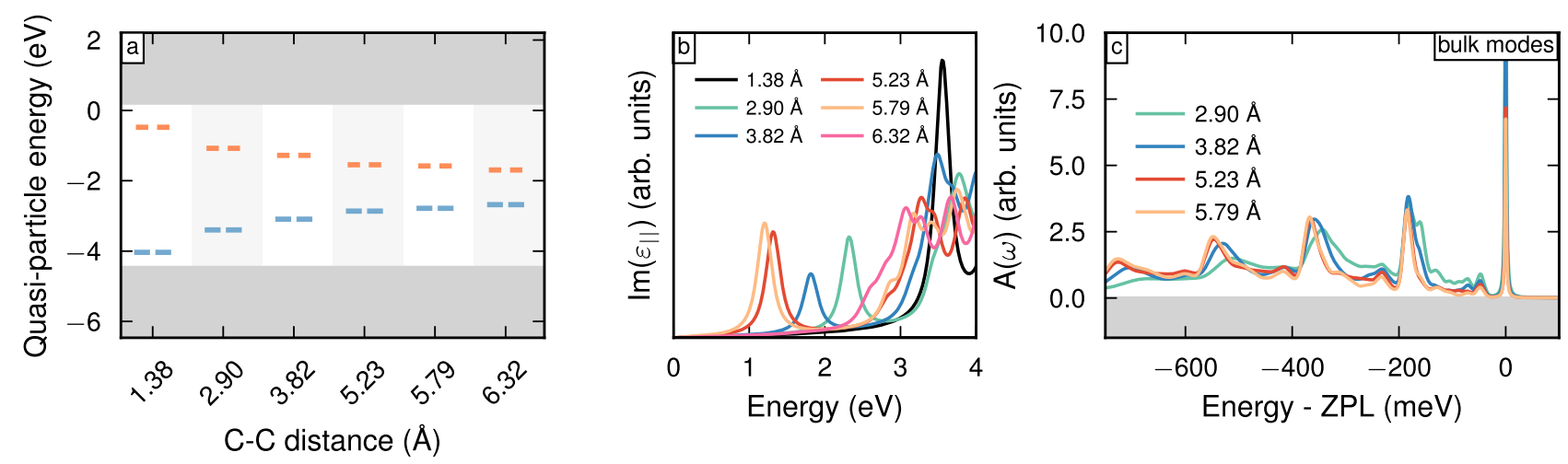

FIG. 7. (a) Kohn-Sham levels for $C_{B}-C_{N}$ defect pairs as a function of separation from PBE calculations. (b) Imaginary part of dielectric function (averaged in plane). (c) Line-shape function for $C_{B}-C_{N}$ pairs.

and $6.32 \AA$. An inspection of the Kohn-Sham levels shows that both LD and LL transitions are possible on the dissociated $\mathrm{C}_{\mathrm{N}}-\mathrm{C}_{\mathrm{B}}$ pair [Fig. 7(a)]. The ZPL for the $\mathrm{LD}+1 / 0$ transition ranges from $2.0 \mathrm{eV}$ to $2.7 \mathrm{eV}$ [see Fig. S3 [41]; all values given here include the band edge shift between PBE and HSE06 $(\alpha=0.6)$ ], while the LL transitions exhibit ZPLs between $2.39 \mathrm{eV}$ at a separation of $2.90 \AA$ and $1.68 \mathrm{eV}$ at a C-C separation of $5.79 \AA$, which is the longest $\mathrm{C}-\mathrm{C}$ distance for which there is a well defined peak in the imaginary part of the dielectric function [Fig. 7(b)].

The vibrational properties of the dissociated $C_{B}-C_{N}$ structures are computationally demanding to obtain due to the low symmetry of the systems. Instead, coupling to bulk phonon modes is considered by utilizing the phonon eigenvectors for the ideal system. Since the lattice distortion induced by $\mathrm{C}_{\mathrm{B}}$ and $\mathrm{C}_{\mathrm{N}}$ is small, the bulk phonon modes are expected to provide a good estimate of the emission line shape. The spectral distribution functions $S(\omega)$ for $\mathrm{C}_{\mathrm{N}}$ computed with ideal modes and defective modes are very similar (see Fig. S2 [41]). This is likely due to the limited structural relaxation relative to the ideal structure that occurs when incorporating $\mathrm{C}$ impurities in h-BN. For defects such as $\mathrm{C}_{\mathrm{B}}-\mathrm{V}_{\mathrm{N}}(\mathrm{T})$ (also shown in Fig. S2 [41]) that significantly distort the lattice, the spectral distribution computed with bulk modes is not a good approximation of the spectral distribution obtained using the modes of the defect structure. This is especially true in cases where the contribution to the HR factor mainly originates from coupling to local (small IPR), defect induced modes.

The spectral distribution functions for the LL transition on dissociated $\mathrm{C}_{\mathrm{B}}-\mathrm{C}_{\mathrm{N}}$ pairs obtained in this fashion are shown in Fig. 7(c) for distances between $2.91 \AA$ and $5.79 \AA . \mathrm{C}_{\mathrm{N}^{-}}$ $\mathrm{C}_{\mathrm{B}}$ defect pairs couple to high frequency bulk modes for all distances considered and have pronounced peaks separated by a frequency of around $187 \mathrm{meV}$ with some minor variations between the different structures.

\section{DISCUSSION}

The only intrinsic defect that emerges from our analysis of the vibrational spectra as a potential $\operatorname{SPE}\left(\mathrm{V}_{\mathrm{B}}\right)$ has a large formation energy. Large formation energies are also obtained for many of the best candidates that are based on extrinsic defects.
The large formation energies are consistent with the observation that pristine $\mathrm{h}$-BN usually exhibits a very small concentration of color centers. To create emitters the h-BN flakes are usually subjected to some treatment, such as electron irradiation [8]. The experimental samples are therefore not equilibrated with respect to the environment, which means that defects with high formation energy may still be present and hence be sensible candidates for color centers. This situation is facilitated by the strong bonding in h-BN, which gives rise to high activation energies for defect migration allowing the stabilization of high-energy defects over very long timescales. As an extreme example, we note the recent preparation of large vacancy clusters [58], the vibrational signatures of which will be the subject of future work.

\section{A. Assignment of defects to the 1.6- to $2.3 \mathrm{eV}$ emitters}

Based on the computed ZPLs $N_{B}-V_{N}, C_{B}-V_{N}, V_{B}^{-}, B_{N}$ and $C_{B}-C_{N}$ pairs could be assigned to the 2-eV emitters (Table I). $\mathrm{N}_{\mathrm{B}}-\mathrm{V}_{\mathrm{N}}$ can be ruled out based on the magnitude of the relaxation between the states involved $\Delta Q$, which leads to an insensibly large $\mathrm{HR}$ factor [2,7]. $\mathrm{B}_{\mathrm{N}}$ can be excluded on the same premises. $C_{B}-V_{N}(T)$, which has been proposed as a candidate [21], is an unlikely source due to the instability of the triplet state. The more stable singlet state, on the other hand, is unlikely to exhibit a structured emission line based on the same argument as for the $\mathrm{N}_{B}-\mathrm{V}_{\mathrm{N}}$ defect.

$\mathrm{V}_{\mathrm{B}}^{-}$has been proposed to be an optically active defect and shown to be able to host transitions in the $2-\mathrm{eV}$ region before [53]. More recently, scanning transmission electron microscopy imaging of vacancies and multivacancies allowed the identification of $\mathrm{V}_{\mathrm{B}}$ defects and led to the association with an emission energy of $1.98 \mathrm{eV}$ [58], which is somewhat higher than the calculated ZPLs of $1.63 \mathrm{eV}\left(C_{2 v}\right)$ and $1.72 \mathrm{eV}\left(D_{3 h}\right.$; Table I). For both $C_{2 v}$ and $D_{3 h}$ configurations, the emission line shape of the HOMO/LUMO transition on $\mathrm{V}_{\mathrm{B}}^{-}$exhibits a rapidly decaying spectral weight away from the ZPL similar to many measured emission spectra in the 2-eV band (Fig. 8). A more detailed comparison with the measured line shape from Ref. [58] [Fig. 8(a)], which has been associated with $V_{B}$, shows, however, that while some features agree between computed and experimental line shapes, such as the position of the first peak close to the ZPL and the peak at around $162 \mathrm{meV}$, which is more pronounced in the case of emission from $D_{3 h}$, 


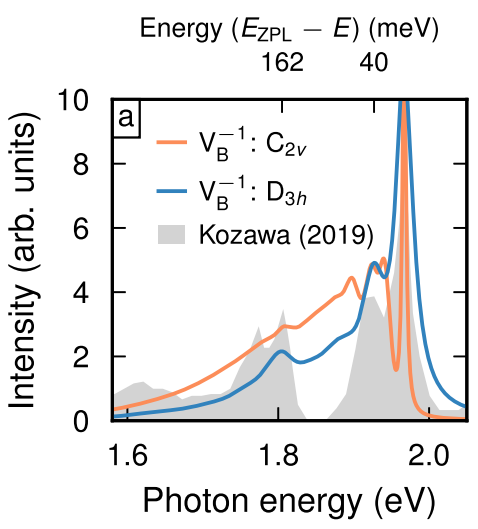

TABLE II. Transition energies and structural displacements from PBE calculations for charge neutral LL transitions on $C_{N}-C_{B}$ defect pairs.

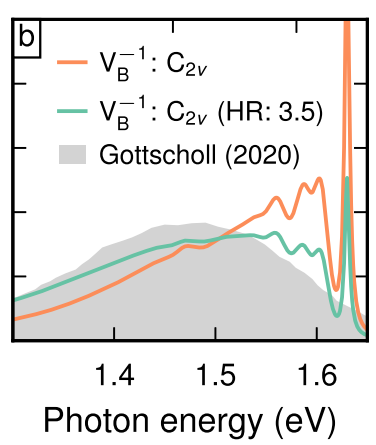

\begin{tabular}{lcc}
\hline \hline Distance $(\AA)$ & ZPL $(\mathrm{eV})$ & $\Delta Q(\sqrt{\mathrm{amu} \AA})$ \\
\hline 1.38 & 3.34 & 0.28 \\
2.90 & 2.39 & 0.42 \\
3.82 & 2.03 & 0.44 \\
5.23 & 1.75 & 0.47 \\
5.79 & 1.68 & 0.46 \\
6.32 & 1.57 & 0.48 \\
\hline \hline
\end{tabular}

FIG. 8. Calculated intensity for the charge neutral transition on $\mathrm{V}_{\mathrm{B}^{*}}^{-}$compared with (a) measurements by Kozawa et al. (Ref. [58]) and (b) measurements by Gottscholl et al. (Ref. [59]). In (a) the measured and computed intensity are both normalized and the computed intensity has been shifted to the measured ZPLs of $1.98 \mathrm{eV}$. The experimental spectrum in Ref. [59] was measured at room temperature. The width of the ZPL has been tuned to match the experimental data from Ref. [58] by setting $\kappa$ in Eq. (7) to $14 \mathrm{meV}$ (3 meV ) for the $D_{3 h}$ $\left(C_{2 v}\right)$ symmetric state.

the overall agreement is far from perfect. Specifically, the ZPL intensity is much larger in the computation and the region between the first peak and second peak carries a large spectral weight in the computation while there is a pronounced gap in most measurements.

On the other hand, more recent measurements of an emitter with a ZPL near $1.6 \mathrm{eV}$ have also been attributed to $\mathrm{V}_{\mathrm{B}}$ [59]. This attribution is not only consistent with the ZPLs calculated here (especially for $C_{2 v}$ ) but has been supported by comprehensive simulations of the room-temperature emission spectrum of $\mathrm{V}_{\mathrm{B}}^{-}$[60]. Both experiment [59] and said simulation [60] show, however, a phonon sideband that exhibits little fine structure and a weak ZPL emission, which is not the case in the present calculations [Fig. 8(b)].

These differences can be understood by recalling the approximations that are made in the present calculations (Sec. IIC), specifically the assumption of low temperature. The intensity of the ZPL often quickly decays with increasing temperature, especially if the phonon side band is broad, which is a signature of large HR factors. This is intuitive as, e.g., phonon-phonon scattering channels naturally lead to broadening and intensity redistribution. Temperature effects were treated at a more comprehensive level in Ref. [60], albeit for only one defect as the approach is computationally substantially more demanding. Indeed the predicted spectrum both features a smoother sideband and a weaker ZPL. The latter study also reports a larger HR factor of 3.5 [60] to be compared with values of $0.9\left(D_{3 h}\right)$ and $2.5\left(C_{2 v}\right)$ obtained in the present study (Table I). This difference is likely due to the use of different exchange-correlation functionals, as hybrid functionals often yield larger HR factors (and values closer to experiment) [29]. In fact, renormalization of the spectral function $S(\omega)$ to a HR factor of 3.5 provides a notably better agreement with the high temperature measurement, especially with regard to the overall width and shape of the sideband [Fig. 8(b)].

Overall, given the approximations made, the present results are consistent with the attribution of $\mathrm{V}_{\mathrm{B}}^{-}$to the emitter at $1.6 \mathrm{eV}$ in accordance with Refs. [59] and [60]. While it is desirable to include temperature effects more comprehensively, doing so at, e.g., the level of Ref. [60], is extensive work and currently not practical for the purpose of screening that is the focus of the present work.

The ZPLs for isolated carbon impurities $\left(\mathrm{C}_{N}, \mathrm{C}_{\mathrm{B}}\right)$ as well as the nearest-neighbor $\mathrm{C}_{B}-\mathrm{C}_{N}$ dimer fall outside of the 1.6to $2.3-\mathrm{eV}$ window considered here. Next-nearest and farther neighbor $\mathrm{C}_{\mathrm{B}}-\mathrm{C}_{\mathrm{N}}$ pairs $(\mathrm{C}-\mathrm{C}$ distance $\geqslant 2.90)$ exhibit, however, varying ZPLs around $2 \mathrm{eV}$ depending on separation distance (Table II). Unlike the (nearest-neighbor) $\mathrm{C}_{\mathrm{B}}-\mathrm{C}_{\mathrm{N}}$ dimer, there are no direct $\mathrm{C}-\mathrm{C}$ bonds present in these configurations and the PSBs mainly originate from coupling to bulk modes. The line shape exhibits only small changes with increasing C-C distance (Fig. 7) while the ZPL varies strongly (Table II), which is a key feature of the SPEs found experimentally in the $2-\mathrm{eV}$ region.

The present analysis demonstrates that dissociated $C_{B}-C_{N}$ defects correspond to a range of different ZPLs with very similar PSBs. In practice, stabilization of these different ZPLs requires confinement of the atoms at specific lattice sites. Since h-BN is a strongly covalent material bond breaking is energetically costly and migration barriers are high. As a result, it is plausible that $C_{B}-C_{N}$ defects can be stabilized at a range of distances.

\section{B. Assignment of defects to the 4.1-eV emitter \\ 1. Isolated carbon impurities $\left(\mathrm{C}_{B}\right.$ and $\left.\mathrm{C}_{N}\right)$}

The 4.1-eV emission line has previously been suggested to originate from $C_{N}[18]$ and carbon-carbon dimers $\left(C_{B}-c n\right.$, Sec. IV B 2) [61]. Focusing first on $C_{N}$, we find that it can host $\mathrm{LD}$ transitions with ZPLs of $4.4 \mathrm{eV}$ (VBM hole capture on $\left.\mathrm{C}_{\mathrm{N}}^{-}\right)$and $2.81 \mathrm{eV}\left(\mathrm{CBM}\right.$ electron capture on $\left.\mathrm{C}_{\mathrm{N}}^{0}\right)$ and moreover demonstrate that $\mathrm{C}_{\mathrm{B}}$ exhibits similar ZPLs. These ZPLs are in rather good agreement with the $4.1-\mathrm{eV}$ line.

The computed line shapes for $C_{N}$ and $C_{B}$ show excellent agreement with the line shape of a $4.1-\mathrm{eV}$ emitter reported in Ref. [17] [Fig. 9(a)]. The frequencies of the dominant modes in $\mathrm{C}_{\mathrm{N}}$ and $\mathrm{C}_{\mathrm{B}}$ are 182 and $187 \mathrm{meV}$, in excellent agreement with the measured frequencies. It is, however, important to note that neither defect distorts the lattice significantly enough to induce local modes, which was argued in Ref. [17] to be the origin of the PSB. We note that the excellent agreement might 
Energy $\left(E_{\mathrm{ZPL}}-E\right)(\mathrm{meV})$ 748561374187

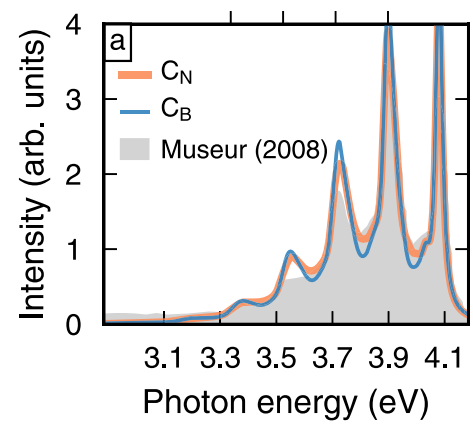

Energy $\left(E_{\mathrm{ZPL}}-E\right)(\mathrm{meV})$ 585390195

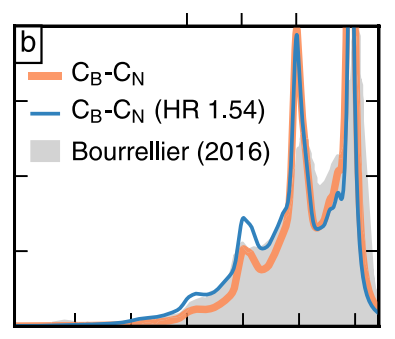

$\begin{array}{llllll}3.1 & 3.3 & 3.5 & 3.7 & 3.9 & 4.1\end{array}$ Photon energy (eV)
FIG. 9. (a) Calculated intensity for the $0 /-1$ transition on $C_{N}$ compared with experimental emission spectrum from Ref. [17]. (b) Calculated intensity for the charge neutral transition on $\mathrm{C}_{\mathrm{B}}-\mathrm{C}_{\mathrm{N}}$ compared with measurements from Ref. [18]. Measured and computed intensity are both normalized and the computed intensity has been shifted to the measured ZPL. The experimental spectra were measured at $9 \mathrm{~K}$ (Ref. [17]) and $150 \mathrm{~K}$ (Ref. [18]), respectively. The width of the ZPL has been tuned to match the experimental data by setting $\kappa$ in Eq. (7) to $14 \mathrm{meV}$.

be partly coincidental since in bulk samples, such as the one measured in Ref. [17], the LO-TO splitting is significant and might push the PSB away from the ZPL by $\sim 10 \mathrm{meV}$. In fact, the highest intensity peak in the PSB for the 4.1-eV emitter has been suggested to originate from coupling to the zone center LO mode at $200 \mathrm{meV}$ [20]. The present calculations for $C_{N}$ and $C_{B}$ yield line shapes and ZPLs consistent with the $4.1 \mathrm{eV}$ emitter observed experimentally.

Our calculations also suggest $C_{B}$ and $C_{N}$ to be strong candidates for emitters found at 3.2 and $3.4 \mathrm{eV}[19,62]$. The line shapes of both $C_{N}$ and $C_{B}$ compare well with the $3.22 \mathrm{eV}$ emitter in Ref. [19], where the first PSB was found at $200 \mathrm{meV}$ from the ZPL with two additional distinct phonon replicas (Fig. 6). The experimental data was recorded at room temperature, which explains the considerably broader spectrum compared to the calculation. The position of the first PSB and the phonon replicas agree well between experiment and calculation safe for a $\sim 10-\mathrm{meV}$ offset, which, as noted above, arises from the presence of LO-TO splitting in a bulk sample. Both ZPLs and line shape thus suggest that $C_{N}$ and $C_{B}$ can be associated with an 3.2-eV emitter.

\section{Carbon-carbon dimer $\left(\mathrm{C}_{B}-\mathrm{C}_{N}\right)$}

As noted above, the 4.1-eV emission has also been associated with the (nearest-neighbor) carbon-carbon dimer $\left(\mathrm{C}_{\mathrm{B}}-\mathrm{C}_{\mathrm{N}}\right)$ [61]. While DFT calculations based on semilocal exchange-correlation (XC) functionals yield a ZPL of $3.34 \mathrm{eV}$ for this defect, hybrid functionals with a larger fraction of exact exchange predict a ZPL in much better agreement with the measured 4.1-eV emission line [61]. Here, it is important to keep in mind that the LL transition involving $C_{B}-C_{N}$ should be much less affected by the band-gap error from the LD transitions on isolated $C_{N}$ or $C_{B}$ since it only involves localized states, which are already well described by semilocal XC functionals.
Our analysis shows that the line shape of the $\mathrm{C}_{B}-\mathrm{C}_{N}$ defect agrees very well with a measured emission spectrum from Ref. [18] [Fig. 9(b)]. The PSBs are located at approximately the correct positions, although the spectral weight of the ZPL is slightly larger for the computed line shape. Interestingly, the $195-\mathrm{meV}$ mode that is the origin of the PSB in the $\mathrm{C}_{\mathrm{B}}-\mathrm{C}_{\mathrm{N}}$ dimer is indeed a local mode in contrast to the dominant modes in $\mathrm{C}_{\mathrm{N}}$ and $\mathrm{C}_{\mathrm{B}}$, which are bulklike. Furthermore, the computed HR factor of 1.1 to 1.2 compares favorably with a measured value of 1.3 for the $4.1-\mathrm{eV}$ emitter [17].

The Stokes shift on the excited state landscape of $0.2 \mathrm{eV}$ obtained with HSE06 $(\alpha=0.25)$ is notably larger than the value of $0.13 \mathrm{eV}$ from PBE. A larger Stokes shift would indicate a smaller spectral weight of the ZPL. Assuming that this difference is dominated by the displacement of the potential energy surfaces relative to each other, the spectral function can be renormalized and, in fact, renormalizing the electronphonon spectral function to a HR factor of 1.54 leads to an even better agreement.

We note that since the PSB originates to a large extent from the local mode at $195 \mathrm{meV}$, which predominantly involves $\mathrm{C}$ motion, isotopic effects may appear in the position of the PSB. This is in contrast to, e.g., the transitions on $C_{N}$ and $C_{B}$, where isotopic control over the $\mathrm{C}$ atoms in the host matrix should not affect the PSB. Hence if one can isotopically control the formation of the $\mathrm{C}_{\mathrm{B}}-\mathrm{C}_{\mathrm{N}}$ dimer to comprise a pair of ${ }^{13} \mathrm{C}$ atoms instead of the naturally occurring ${ }^{12} \mathrm{C}$ one should detect a small variation in the position of the PSB if the $4.1 \mathrm{eV}$ line originates from the $C_{B}-C_{N}$ dimer. Based on our calculations we estimate this frequency shift to be $4 \mathrm{meV}$, placing the local $\mathrm{C}$-C mode at $191 \mathrm{meV}$ for ${ }^{13} \mathrm{C}$. While emitters based on ${ }^{13} \mathrm{C}$ have been fabricated [63], changes in the PSB have not been investigated yet.

The transitions on intrinsic defects that have ZPLs in the vicinity of $4.1 \mathrm{eV}$ include hole capture on $\mathrm{V}_{\mathrm{N}}^{0}$, as well as hole capture on $\mathrm{V}_{\mathrm{B}}^{-}$, which give rise to ZPLs of $4.04 \mathrm{eV}$ and $4.12 \mathrm{eV}$, respectively (see Table I). However, the structural distortions associated with these charged (LD) transitions are relatively large and unlikely to be compatible with the measured line shape and HR factor (Table I).

\section{CONCLUSIONS}

We have analyzed the optical transitions occurring from charge transitions (LD) and charge neutral (LL) transitions for a wide range of defects in h-BN. For a selection of these defects, we have examined the possibility of assigning defect emission to well known SPEs by calculating line shapes and emission spectra with the generating function method. We have found that $V_{B}, C_{N}, C_{B}$, and $C_{B}-C_{N}$ can host transitions that couple strongly to high-frequency modes while still exhibiting a moderate HR factor between 0.9 and 2.5.

The main conclusions are as follows:

(i) The line shapes of $C_{N}$ and $C_{B}$ are in excellent agreement with measured line shapes for the 4.1-eV emitter. Furthermore, the ZPLs are in good agreement with the $4.1 \mathrm{eV}$ ZPL. In addition, we note that these defects also exhibit ZPLs for the reverse transition that agrees well with the observed 3.2- to 3.4-eV luminescence. 
(ii) Next-nearest and farther neighbor (dissociated) $C_{B}-C_{N}$ defect pairs allow for both charge neutral and charged transitions over a wide range of ZPL energies with narrow emission bands.

(iii) Our findings support the assignment of the $4.1 \mathrm{eV}$ emission to the $C_{B}-C_{N}$ dimer (nearest-neighbor pair) based on the line shape but we note that there are additional defects that exhibit similar emission line shapes.

(iv) Depending on the symmetry of the excited state of $\mathrm{V}_{\mathrm{B}}^{-}$(and temperature), the emission can be either focused in a narrow energy range around the ZPL $\left(D_{3 h}\right)$ or have a wider phonon sideband $\left(C_{2 v}\right)$.

The current results indicate that out of the emitters considered, $\mathrm{V}_{\mathrm{B}}$ and $\mathrm{C}$ dimers at intermediate distance are the best candidates for emission in the low frequency band (1.6 to $2.3 \mathrm{eV}$ ) while isolated $\mathrm{C}$ impurities as well as nearestneighbor $\mathrm{C}$ dimers are the best candidates for the high frequency $(\sim 4.1-\mathrm{eV})$ emitter(s). We note that recently additional defect configurations have been suggested that have not been considered here, including Stone-Wales defects [64] as well as oxygen-carbon pairs [65].

Finally, we want to emphasize that for the defects considered in this study, it is nontrivial to generate the excited state at the hybrid level, due to the presence of a series of localized levels in the band gap (Sec. IID). This points to the need of further investigations into the correct description of the subtleties of the electronic structure of these defects. As evident, in particular from the analysis of the $V_{B}$ defect (Sec. IV A) and the comparison with Ref. [60], it can moreover, be useful or even necessary to include temperature effects more comprehensively when targeting specific defects.

\section{ACKNOWLEDGMENTS}

We are grateful to Arsalan Hashemi and Hannu-Pekka Komsa for fruitful discussions and for providing us with the finite-size corrections for the calculations of the defect formation energies in Ref. [38]. We acknowledge the Knut and Alice Wallenberg Foundation (Grants No. 2014.0226 and No. 2019.0231), the Swedish Research Council (Grants No. 2018-06482 and No. 2020-04935), and the Chalmers Excellence Initiative Nano for financial support. The computations were enabled by resources provided by the Swedish National Infrastructure for Computing (SNIC) at NSC, C3SE, and PDC partially funded by the Swedish Research Council through Grant No. 2018-05973.
[1] X. Z. Du, J. Li, J. Y. Lin, and H. X. Jiang, Appl. Phys. Lett. 108, 052106 (2016).

[2] T. T. Tran, K. Bray, M. J. Ford, M. Toth, and I. Aharonovich, Nat. Nanotechnol. 11, 37 (2015).

[3] J. L. O'Brien, A. Furusawa, and J. Vukovi, Nat. Photon. 3, 687 (2009).

[4] I. Aharonovich, D. Englund, and M. Toth, Nat. Photon. 10, 631 (2016).

[5] T. T. Tran, M. Kianinia, M. Nguyen, S. Kim, Z.-Q. Xu, A. Kubanek, M. Toth, and I. Aharonovich, ACS Photon. 5, 295 (2018).

[6] A. L. Exarhos, D. A. Hopper, R. R. Grote, A. Alkauskas, and L. C. Bassett, ACS Nano 11, 3328 (2017).

[7] Z. Shotan, H. Jayakumar, C. R. Considine, M. Mackoit, H. Fedder, J. Wrachtrup, A. Alkauskas, M. W. Doherty, V. M. Menon, and C. A. Meriles, ACS Photon. 3, 2490 (2016).

[8] T. T. Tran, C. Elbadawi, D. Totonjian, C. J. Lobo, G. Grosso, H. Moon, D. R. Englund, M. J. Ford, I. Aharonovich, and M. Toth, ACS Nano 10, 7331 (2016).

[9] D. Wigger, R. Schmidt, O. D. Pozo-Zamudio, J. A. Preu, P. Tonndorf, R. Schneider, P. Steeger, J. Kern, Y. Khodaei, J. Sperling, S. M. de Vasconcellos, R. Bratschitsch, and T. Kuhn, 2D Mater. 6, 035006 (2019).

[10] A. Bommer and C. Becher, Nanophotonics 8, 2041 (2019).

[11] A. Dietrich, M. W. Doherty, I. Aharonovich, and A. Kubanek, Phys. Rev. B 101, 081401(R) (2020).

[12] M. Atatüre, D. Englund, N. Vamivakas, S.-Y. Lee, and J. Wrachtrup, Nat. Rev. Mater. 3, 38 (2018).

[13] M. Abdi, M.-J. Hwang, M. Aghtar, and M. B. Plenio, Phys. Rev. Lett. 119, 233602 (2017).

[14] M. Abdi and M. B. Plenio, Phys. Rev. Lett. 122, 023602 (2019).

[15] Y. Xia, Q. Li, J. Kim, W. Bao, C. Gong, S. Yang, Y. Wang, and X. Zhang, Nano Lett. 19, 7100 (2019).
[16] N. Nikolay, N. Mendelson, E. Özelci, B. Sontheimer, F. Böhm, G. Kewes, M. Toth, I. Aharonovich, and O. Benson, Optica 6, 1084 (2019).

[17] L. Museur, E. Feldbach, and A. Kanaev, Phys. Rev. B 78, 155204 (2008).

[18] R. Bourrellier, S. Meuret, A. Tararan, O. Stéphan, M. Kociak, L. H. G. Tizei, and A. Zobelli, Nano Lett. 16, 4317 (2016).

[19] E. Tsushima, T. Tsujimura, and T. Uchino, Appl. Phys. Lett. 113, 031903 (2018).

[20] T. Q. P. Vuong, G. Cassabois, P. Valvin, A. Ouerghi, Y. Chassagneux, C. Voisin, and B. Gil, Phys. Rev. Lett. 117, 097402 (2016).

[21] S. A. Tawfik, S. Ali, M. Fronzi, M. Kianinia, T. T. Tran, C. Stampfl, I. Aharonovich, M. Toth, and M. J. Ford, Nanoscale 9, 13575 (2017).

[22] L. Weston, D. Wickramaratne, M. Mackoit, A. Alkauskas, and C. G. Van de Walle, Phys. Rev. B 97, 214104 (2018).

[23] M. A. Feldman, A. Puretzky, L. Lindsay, E. Tucker, D. P. Briggs, P. G. Evans, R. F. Haglund, and B. J. Lawrie, Phys. Rev. B 99, 020101(R) (2019).

[24] G. Grosso, H. Moon, C. J. Ciccarino, J. Flick, N. Mendelson, L. Mennel, M. Toth, I. Aharonovich, P. Narang, and D. R. Englund, ACS Photon. 7, 1410 (2020).

[25] J. J. Markham, Rev. Mod. Phys. 31, 956 (1959).

[26] We adhere to the convention that the cohesive energy is defined as the energy gained on formation from the atomic states and hence commonly a positive quantity.

[27] M. Lax, J. Chem. Phys. 20, 1752 (1952).

[28] R. Kubo and Y. Toyozawa, Prog. Theor. Phys. 13, 160 (1955).

[29] A. Alkauskas, B. B. Buckley, D. D. Awschalom, and C. G. V. de Walle, New J. Phys. 16, 073026 (2014).

[30] R. Borrelli, A. Capobianco, and A. Peluso, J. Phys. Chem. A 116, 9934 (2012). 
[31] A. Alkauskas, J. L. Lyons, D. Steiauf, and C. G. Van de Walle, Phys. Rev. Lett. 109, 267401 (2012).

[32] G. Kresse and J. Furthmüller, Phys. Rev. B 54, 11169 (1996).

[33] P. E. Blöchl, Phys. Rev. B 50, 17953 (1994).

[34] G. Kresse and D. Joubert, Phys. Rev. B 59, 1758 (1999).

[35] G. Kresse and J. Hafner, Phys. Rev. B 47, 558 (1993).

[36] J. P. Perdew, K. Burke, and M. Ernzerhof, Phys. Rev. Lett. 77, 3865 (1996).

[37] J. Heyd, G. E. Scuseria, and M. Ernzerhof, J. Chem. Phys. 118, 8207 (2003).

[38] H.-P. Komsa, N. Berseneva, A. V. Krasheninnikov, and R. M. Nieminen, Phys. Rev. X 4, 031044 (2014).

[39] H.-P. Komsa (private communication).

[40] A. Togo and I. Tanaka, Scr. Mater. 108, 1 (2015).

[41] See Supplemental Material at http://link.aps.org/supplemental/ 10.1103/PhysRevB.103.115421 for additional figures of the spectral and lineshape functions and of figures pertaining to the electronic state analysis.

[42] W. Paszkowicz, J. Pelka, M. Knapp, T. Szyszko, and S. Podsiadlo, Appl. Phys. A 75, 431 (2002).

[43] S. Haastrup, M. Strange, M. Pandey, T. Deilmann, P. S. Schmidt, N. F. Hinsche, M. N. Gjerding, D. Torelli, P. M. Larsen, A. C. Riis-Jensen, J. Gath, K. W. Jacobsen, J. J. Mortensen, T. Olsen, and K. S. Thygesen, 2D Mater. 5, 042002 (2018).

[44] C. Elias, P. Valvin, T. Pelini, A. Summerfield, C. J. Mellor, T. S. Cheng, L. Eaves, C. T. Foxon, P. H. Beton, S. V. Novikov, B. Gil, and G. Cassabois, Nat. Commun. 10, 2639 (2019).

[45] G. Cassabois, P. Valvin, and B. Gil, Nat. Photon. 10, 262 (2016).

[46] B. Arnaud, S. Lebègue, P. Rabiller, and M. Alouani, Phys. Rev. Lett. 96, 026402 (2006).

[47] R. Tutchton, C. Marchbanks, and Z. Wu, Phys. Rev. B 97, 205104 (2018).

[48] Since LO-TO splitting is absent in 2D materials [66], the nonanalytical contribution to the force constant matrix has been omitted.

[49] J. Carrete, W. Li, L. Lindsay, D. A. Broido, L. J. Gallego, and N. Mingo, Mater. Res. Lett. 4, 204 (2016).
[50] J. Serrano, A. Bosak, R. Arenal, M. Krisch, K. Watanabe, T. Taniguchi, H. Kanda, A. Rubio, and L. Wirtz, Phys. Rev. Lett. 98, 095503 (2007).

[51] T. Tohei, A. Kuwabara, F. Oba, and I. Tanaka, Phys. Rev. B 73, 064304 (2006).

[52] J. R. Reimers, A. Sajid, R. Kobayashi, and M. J. Ford, J. Chem. Theory Comput. 14, 1602 (2018).

[53] M. Abdi, J.-P. Chou, A. Gali, and M. B. Plenio, ACS Photon. 5, 1967 (2018).

[54] M. R. Uddin, J. Li, J. Y. Lin, and H. X. Jiang, Appl. Phys. Lett. 110, 182107 (2017).

[55] A. Sajid, J. R. Reimers, and M. J. Ford, Phys. Rev. B 97, 064101 (2018).

[56] F. Wu, A. Galatas, R. Sundararaman, D. Rocca, and Y. Ping, Phys. Rev. Materials 1, 071001(R) (2017).

[57] G. Noh, D. Choi, J.-H. Kim, D.-G. Im, Y.-H. Kim, H. Seo, and J. Lee, Nano Lett. 18, 4710 (2018).

[58] D. Kozawa, A. G. Rajan, S. X. Li, T. Ichihara, V. B. Koman, Y. Zeng, M. Kuehne, S. K. Iyemperumal, K. S. Silmore, D. Parviz, P. Liu, A. T. Liu, S. Faucher, Z. Yuan, W. Xu, J. H. Warner, D. Blankschtein, and M. S. Strano, arXiv:1909.11738.

[59] A. Gottscholl, M. Kianinia, V. Soltamov, S. Orlinskii, G. Mamin, C. Bradac, C. Kasper, K. Krambrock, A. Sperlich, M. Toth, I. Aharonovich, and V. Dyakonov, Nat. Mater. 19, 540 (2020).

[60] V. Ivády, G. Barcza, G. Thiering, S. Li, H. Hamdi, J.-P. Chou, Ö. Legeza, and A. Gali, npj Comput. Mater. 6, 41 (2020).

[61] M. Mackoit-Sinkevičienè, M. Maciaszek, C. G. Van de Walle, and A. Alkauskas, Appl. Phys. Lett. 115, 212101 (2019).

[62] B. Berzina, V. Korsaks, L. Trinkler, A. Sarakovskis, J. Grube, and S. Bellucci, Diam. Relat. Mater. 68, 131 (2016).

[63] T. Pelini, C. Elias, R. Page, L. Xue, S. Liu, J. Li, J. H. Edgar, A. Dréau, V. Jacques, P. Valvin, B. Gil, and G. Cassabois, Phys. Rev. Materials 3, 094001 (2019).

[64] H. Hamdi, G. Thiering, Z. Bodrog, V. Ivády, and A. Gali, npj Comput. Mater. 6, 178 (2020).

[65] A. S. Vokhmintsev and I. A. Weinstein, arXiv:2003.02789.

[66] T. Sohier, M. Gibertini, M. Calandra, F. Mauri, and N. Marzari, Nano Lett. 17, 3758 (2017). 